\title{
Stability of Strong Tracking Filter with Markovian Packet Losses
}

\author{
Chen Xiaocen \\ Department of Optical and Electrical Engineering, Ordnance Engineering College, \\ Shijiazhuang, 050003, China
}

\begin{abstract}
We consider the stability of strong tracking filter (STF) in the networked control system. First, we study the stability of strong tracking filter for systems with colored observation noises using augmentation and a theorem of bounded stochastic functions. Then by analyzing the error covariance of STF in different network environment, we prove the boundedness of the peak error covariance.
\end{abstract}

Keywords -Strong tracking filter, Nonlinear system, Colored noises, Networked control system, Peak error covariance.

\section{INTRODUCTION}

In the recent years, networked control systems have gained more and more attention from both the communication filed and control filed. It has very broad application from navigation to environment monitoring. The reason for this phenomenon is due to several advantages of the system. It can increase the speed and agility of the system, reduce the system wiring and monitor the system easily. However, it also has some disadvantages such as the packet delayed and dropped which affect the accuracy of the transmitted information. This makes it necessary to calculate the peak error covariance.

Many researchers study the error covariance of the linear time invariant system in the networked control system. [1] studied error covariance from the expectation angel and concluded the bound of the packet arrival rate, below which, the expectation of error covariance would go infinity. [2] considered the issue in a probabilistic perspective $\operatorname{Pr}[\mathrm{Pk}<\mathrm{M}]$ and extended the result of [1]. [3] concluded a necessary and sufficient condition for the stability of discrete-time networked control system using pole placement.

Most studies are about the linear networked control system using Kalman filtering. However, in reality, many systems are nonlinear. Moreover, strong tracking filter is superior to Kalman filter in many aspects, for example it can adapt the abrupt changes and track the system better, it has self-accommodation to the inaccuracy of the model as well as parameters. The contribution of this paper is that we put forward nonlinear issue in the networked control system using strong tracking filtering.

\section{PROBLEM FORMULATION}

We consider the following nonlinear system with colored noises

$$
\left\{\begin{array}{c}
x_{k+1}=f\left(k, u_{k}, x_{k}\right)+\Gamma_{k} v_{k} \\
y_{k+1}=h\left(k+1, x_{k+1}\right)+B_{k+1} e_{k+1}+D_{k+1} \xi_{k+1}
\end{array}\right.
$$

where $v_{k}, \xi_{k}$ are Gaussian white noises, and $e_{k}$ is colored observation noise

$$
\left\{\begin{array}{c}
E\left(v_{k}\right)=0, \operatorname{cov}\left(v_{k}, v_{j}\right)=R_{k} \delta_{k, j} \\
E\left(\xi_{k}\right)=0, \operatorname{cov}\left(\xi_{k}, \xi_{j}\right)=S_{k} \delta_{k, j} . \\
\operatorname{cov}\left(v_{k}, \xi_{j}\right)=0
\end{array}\right.
$$

We decompose the nonlinear function $f\left(k, u_{k}, x_{k}\right), h\left(k+1, x_{k+1}\right)$ by Taylor expansion.

$$
\begin{aligned}
& f\left(k, u_{k}, x_{k}\right)=f\left(k, u_{k}, \hat{x}_{k}\right)+F_{k}\left(x_{k}-\hat{x}_{k}\right)+\varphi\left(x_{k}, \hat{x}_{k}, k\right) \\
& h\left(k, x_{k}\right)=h\left(k, \hat{x}_{k}\right)+H_{k}\left(x_{k}-\hat{x}_{k}\right)+\chi\left(x_{k}, \hat{x}_{k}, k\right)
\end{aligned}
$$

The nonlinear system can be simplified as linear time-variant system

$$
\begin{aligned}
& \left\{\begin{array}{c}
x_{k+1}=F_{k} x_{k}+t_{k}+\Gamma_{k} v_{k} \\
y_{k}=H_{k} x_{k}+B_{k} e_{k}+D_{k} \xi_{k}+m_{k}
\end{array}\right. \\
& t_{k}=f\left(k, u_{k}, x_{k}\right)-F_{k} \hat{x}_{k}+\varphi\left(x_{k}, \hat{x}_{k}, k\right), m_{k}=h\left(k, x_{k}\right)-H_{k} \hat{x}_{k}+\chi\left(x_{k}, \hat{x}_{k}, k\right)
\end{aligned}
$$

To remove the colored noise, we use augmentation to simplify the system. Here we define

$$
\begin{aligned}
& \left\{\begin{array}{c}
\alpha_{k+1}=\bar{A} \alpha_{k}+\bar{C} \varepsilon_{k}, E\left(\varepsilon_{k}\right)=0 \\
e_{k}=\bar{B} \alpha_{k}
\end{array}\right. \\
& Z_{k}=\left[x_{k}^{T}, \alpha_{k}^{T}\right]^{T}, w_{k}=\left[v_{k}^{T}, \varepsilon_{k}^{T}\right], T_{k}=\left[\begin{array}{c}
t_{k} \\
0
\end{array}\right], A_{k}=\left[\begin{array}{cc}
F_{k} & 0 \\
0 & \bar{A}
\end{array}\right], L_{k}=\left[\begin{array}{cc}
\Gamma_{k} & 0 \\
0 & \bar{C}
\end{array}\right], E_{k}=\left[H_{k}, B_{k} \bar{B}\right]
\end{aligned}
$$

Then we get the augmented state and observation equations:

$$
\left\{\begin{array}{l}
Z_{k+1}=A_{k} Z_{k}+L_{k} w_{k}+T_{k} \\
y_{k}=E_{k} Z_{k}+D_{k} \xi_{k}+m_{k}
\end{array}\right.
$$

According to Zhou D.H in [4], the algorithm of strong tracking filter is as follows:

$$
\left\{\begin{array}{c}
P_{k}=\left[I-K_{k} E_{k}\right] P_{k}^{-} \\
P_{k+1}^{-}=\lambda_{k+1} A_{K} P_{k} A_{k}^{T}+Q_{k} \\
K_{k}=P_{k}^{-} E_{k}\left[E_{k} P_{k}^{-} E_{k}^{T}+S_{k}\right]^{-1}
\end{array}\right.
$$

where $\lambda_{k+1}=\left\{\begin{array}{ll}\lambda_{0}, & \lambda_{0} \geq 1 \\ 1, & \lambda_{0}<1\end{array}\right.$, and

$\left\{\begin{array}{l}\lambda_{0}=\operatorname{tr}\left[N_{k+1} / M_{k+1}\right] \\ N_{k+1}=\bar{V}_{k+1}-E_{k+1} Q_{k} E_{k+1}^{T}-S_{k+1}, M_{k+1}=E_{k+1} A_{k} P_{K} A_{k}^{T} E_{k}^{T} . \\ \bar{V}_{k+1}=E_{k+1} P_{k+1}^{-} E_{k+1}^{T}+S_{k+1}\end{array}\right.$

Then we can get the following recursive algorithm of error covariance:

$P_{k+1}=\lambda_{k+1} A_{k} P_{k} A_{k}^{T}-\lambda_{k+1} A_{k} P_{k} E_{k}^{T}\left(E_{k} P_{k} E_{k}^{T}+S_{k}\right) E_{k} P_{k} A_{k}^{T}+Q_{k}$ 


\section{MAIN RESULTS}

When the packet arrival rate $\gamma_{t}=1$, we have the bound of the error covariance matrix of strong tracking filter for the nonlinear system with colored observation noises.

Condition A1:

$\left\|A_{k}\right\| \leq \bar{a},\left\|L_{k}\right\| \leq \bar{L},\left\|E_{k}\right\| \leq \bar{e},\left\|D_{k}\right\| \leq \bar{d},\left\|Q_{k}\right\| \leq \bar{q}, \underline{p} I \leq P_{k} \leq \bar{p} I, S_{k} \geq \underline{s} I$

\section{Theorem 1}

Under condition $\mathrm{A} 1, \quad P_{k} \leq d_{k}^{(1)} P_{0}+d_{k}^{(2)}$, as well as $P_{k+1}<P_{k}$, that is to say the error covariance matrix is decreasing.

To prove the theorem, we introduce 2 lemmas.

Lemma 1: Assume there is a stochastic process $V_{n}\left(\varsigma_{n}\right)$ as well as real numbers $\bar{v}, \underline{v}, \mu>0$ and $0<\alpha \leq 1$ such that

$$
\underline{v}\left\|\varsigma_{n}\right\|^{2} \leq V_{n}\left(\varsigma_{n}\right) \leq \bar{v}\left\|\varsigma_{n}\right\|^{2} \quad, \quad \text { and }
$$

$E\left\{V_{n+1}\left(\varsigma_{n+1}\right) \mid \varsigma_{n}\right\}-V_{n}\left(\varsigma_{n}\right) \leq \mu-\alpha V_{n}\left(\varsigma_{n}\right)$

Then the stochastic process is exponentially bounded in mean square. We have

$$
E\left\{\left\|\varsigma_{n}\right\|^{2}\right\} \leq \frac{\bar{v}}{\underline{v}} E\left\{\left\|\varsigma_{0}\right\|^{2}\right\}(1-\alpha)^{n}+\frac{\mu}{\underline{\mu}} \sum_{i=1}^{n-1}(1-\alpha)^{i}
$$

Moreover, the stochastic process is bounded with probability one. [5]

Lemma 2: There exits a real number $0<\alpha \leq 1$, such that $\Pi_{n}=P_{n}^{-1}$ satisfies the inequality

$$
\left(A_{k}-K_{k} E_{k}\right)^{T} \Pi_{n+1}\left(A_{k}-K_{k} E_{k}\right) \leq(1-\alpha) \Pi_{n}
$$

The proof is similar as that in [5]

$$
\begin{aligned}
& P_{k+1}=\lambda_{k+1}\left(A_{k}-K_{k} E_{k}\right) P_{k}\left(A_{k}-K_{k} E_{k}\right)^{T}+Q_{k} \\
& +\lambda_{k+1} K_{k} E_{k} P_{k}\left(A_{k}-K_{k} E_{k}\right)^{T} \\
& \text { As } \\
& \lambda_{k+1} K_{k} E_{k} P_{k} \\
& =\lambda_{k+1} A_{k}\left[A_{k}^{-} K_{k} E_{k}\right]\left[A_{k}^{-}\left(A_{k}-K_{k} E_{k}\right) P_{k}\right]^{T} A_{k}^{T}
\end{aligned}
$$

We can study the two parts $A_{k}^{-} K_{k} E_{k}$,

$A_{k}^{-}\left(A_{k}-K_{k} E_{k}\right) P_{k}$ respectively.

$$
\begin{aligned}
& {\left[A_{k}^{-}\left(A_{k}-K_{k} E_{k}\right) P_{k}\right]} \\
& =P_{k}-P_{k} E_{k}^{T}\left(E_{k} P_{k} E_{k}^{T}+R_{k}\right)^{-1} E_{k} P_{k}
\end{aligned}
$$

From the matrix inversion lemma,

$$
\begin{aligned}
& {\left[A_{k}^{-}\left(A_{k}-K_{k} E_{k}\right) P_{k}\right]} \\
& =\left(P_{k}^{-}+E_{k}^{T} R_{k}^{-} E_{k}\right)^{-1}>0 \\
& A_{k}^{-} K_{k} E_{k}=P_{k} E_{k}^{T}\left(E_{k} P_{k} E_{k}^{T}+R_{k}\right)^{-1} E_{k}>0
\end{aligned}
$$

Meanwhile, from Zhou., we conclude

$$
\lambda_{k+1}=\operatorname{trace}\left(N_{k+1} / M_{k+1}\right)>0
$$

So,

$$
\begin{aligned}
& \lambda_{k+1} K_{k} E_{k} P_{k} \\
& =\lambda_{k+1} A_{k}\left[A_{k}^{-} K_{k} E_{k}\right]\left[A_{k}^{-}\left(A_{k}-K_{k} E_{k}\right) P_{k}\right]^{T} A_{k}^{T}>0
\end{aligned}
$$

Next, we come to the equation:

$$
\begin{aligned}
& P_{k+1}=\lambda_{k+1}\left(A_{k}-K_{k} E_{k}\right) P_{k}\left(A_{k}-K_{k} E_{k}\right)^{T}+Q_{k} \\
& +\lambda_{k+1} K_{k} E_{k} P_{k}\left(A_{k}-K_{k} E_{k}\right)^{T} \\
& \geq \lambda_{k+1}\left(A_{k}-K_{k} E_{k}\right) P_{k}\left(A_{k}-K_{k} E_{k}\right)^{T}+Q_{k} \\
& =\lambda_{k+1}\left(A_{k}-K_{k} E_{k}\right)^{*} \\
& {\left[P_{k}+\frac{1}{\lambda_{k+1}}\left(A_{k}-K_{k} E_{k}\right)^{-1} Q_{k}\left(A_{k}-K_{k} E_{k}\right)^{-T}\right]\left(A_{k}-K_{k} E_{k}\right)^{T}}
\end{aligned}
$$

then inversing the two side of the upper equation, we can get the following relationship:

$$
\left(A_{k}-K_{k} E_{k}\right)^{T} \Pi_{k+1}\left(A_{k}-K_{k} E_{k}\right)
$$$$
\leq \frac{\Pi_{k}}{\lambda_{k+1}}\left[1+\frac{1}{\lambda_{k+1}}\left(A_{k}-K_{k} E_{k}\right)^{-1} Q_{k}\left(A_{k}-K_{k} E_{k}\right)^{-T} \Pi_{k}\right]^{-1}
$$

Which completes the proof

$$
1-\alpha=\frac{1}{\lambda_{k+1}}\left[1+\frac{1}{\lambda_{k+1}} \frac{\underline{q}}{\bar{p}\left(\bar{a}+\frac{\overline{a p e}^{2}}{\underline{s}}\right)^{2}}\right]^{-1}
$$

Now we come to the state and observation equation as follows:

$$
\left\{\begin{array}{c}
Z_{k+1}=A_{k} Z_{k}+L_{k} w_{k}+T_{k} \\
y_{k}=E_{k} Z_{k}+D_{k} \xi_{k}+m_{k}
\end{array}\right.
$$

Based on the Kalman filtering, we can get

$$
\hat{Z}_{k+1}=A_{k} \hat{Z}_{k}+T_{k}+K_{k}\left(y_{k}-E_{k} \hat{Z}_{k}-m_{k}\right)
$$

As $\Delta_{k}=z_{k}-\hat{z}_{k}$, we can yield

$\Delta_{k+1}=\left(A_{k}-K_{k} E_{k}\right) \Delta_{k}+L_{k} w_{k}-K_{k} D_{k} \xi_{k}$

We define $V_{n}\left(\varsigma_{n}\right)=\varsigma_{n}^{T} \Pi_{n} \varsigma_{n}$

$$
\begin{aligned}
& V_{n+1}\left(S_{n+1}\right)=\Delta_{k}^{T}\left(A_{k}-K_{k} E_{k}\right)^{T} \Pi_{n+1}\left(A_{k}-K_{k} E_{k}\right) \Delta_{k}+w_{k}^{T} L_{k}^{T} \Pi_{n+1}\left[2\left(A_{k}-K_{k} E_{k}\right) \Delta_{k}\right] \\
& -2 \xi_{k}^{T} D_{k}^{T} K_{k}^{T} \Pi_{n+1} L_{k} w_{k}-\xi_{k}^{T} D_{k}^{T} K_{k}^{T} \Pi_{n+1}\left[2\left(A_{k}-K_{k} E_{k}\right) \Delta_{k}\right]+w_{k}^{T} L_{k}^{T} \Pi_{n+1} L_{k} w_{k}+\xi_{k}^{T} D_{k}^{T} K_{k}^{T} \Pi_{n+1} K_{k} D_{k} \xi_{k}
\end{aligned}
$$

As $\xi_{k}$ is independent of $\Pi_{n+1}, \Delta_{k}$, so the expectation of $\xi_{k}^{T} D_{k}^{T} K_{k}^{T} \Pi_{n+1} L_{k} w_{k}$ and

$\xi_{k}^{T} D_{k}^{T} K_{k}^{T} \Pi_{n+1}\left[2\left(A_{k}-K_{k} E_{k}\right) \Delta_{k}\right]$ is zero.

We assume $E\left(w_{k} w_{k}^{T}\right)=w I, \operatorname{tr}\left(L_{k} L_{k}^{T}\right) \leq \Upsilon$

Then $E\left(w_{k}^{T} L_{k}^{T} \Pi_{n+1} L_{k} w_{k}\right) \leq \frac{w \Upsilon}{\underline{p}}$. 
Similarly,we

assume $\quad E\left(\xi_{k} \xi_{k}^{T}\right)=\xi I, \operatorname{tr}\left(K_{k} D_{k}\left(K_{k} D_{k}\right)^{T}\right) \leq m$

then $E\left(\xi_{k}^{T} D_{k}^{T} K_{k}^{T} \Pi_{n+1} K_{k} D_{k} \xi_{k}\right) \leq \frac{\xi m}{p}$.

According to lemma 1, we have the following conclusion

$$
P_{k} \leq \frac{\bar{p}}{\underline{p}}\left\|P_{0}\right\|(1-\alpha)^{k}+\bar{p}\left(\frac{w \Upsilon}{\underline{p}}+\frac{\xi m}{\underline{p}}\right) \sum_{i=1}^{k-1}(1-\alpha)^{i} .
$$

We can simplify the inequality as

$P_{k} \leq A(1-\alpha)^{k}+B \sum_{i=1}^{k-1}(1-\alpha)^{i}(A>0, B>0)$.

Accordingly,

$$
P_{k+1}-P_{k} \leq-A \alpha(1-\alpha)^{k}-B \alpha \sum_{i=1}^{k-1}(1-\alpha)^{i}, P_{k+1} \leq P_{k}+C_{0} \text {. }
$$

which completes the proof of theorem 1 .

In the networked control system, we consider the packet arrival rate $\gamma_{t}=1$ if we get the packet successfully and $\gamma_{t}=0$ if the packet is lost.

$\gamma_{t}=1, P_{k+1}=\lambda_{k+1} A_{k} P_{k} A_{k}^{T}-\lambda_{k+1} A_{k} P_{k} E_{k}^{T}\left(E_{k} P_{k} E_{k}^{T}+S_{k}\right) E_{k} P_{k} A_{k}^{T}+Q_{k}$ $\gamma_{t}=0, P_{k+1}=A_{k} P_{k} A_{k}^{T}+Q_{k}$

According to [6], the author defines $a_{k}$ as the kth packet loss after successive packet arrivals and $b_{k}$ as the kth packet arrival after successive packet losses.

$$
\begin{aligned}
& a_{i}^{*}=a_{i}-b_{i-1}, b_{i}^{*}=b_{i}-a_{i} \\
& P\left(a_{i}^{*}-1=k\right)=(1-p)^{k} p, P\left(b_{i}^{*}-1=k\right)=(1-q)^{k} q
\end{aligned}
$$

([6], lemma2)

Now we calculate the bound of the peak error covariance matrix

$$
P_{b_{k}}\left(P_{b_{k}}=P_{b_{k} \mid b_{k-1}}\right) \text {. }
$$

Theorem 2

We assume condition

$A 2:\left|\bar{a}^{2}(1-q)\right|<1$, under which we can draw the conclusion that the peak error covariance matrix is bounded.

Proof:

At first, we define

$F_{K}\left(P_{K}\right)=P_{k+1}=\lambda_{k+1} A_{k} P_{k} A_{k}^{T}-\lambda_{k+1} A_{k} P_{k} E_{k}^{T}\left(E_{k} P_{k} E_{k}^{T}+S_{k}\right) E_{k} P_{k} A_{k}^{T}+Q_{k}$

$$
\phi(i, j)=F_{i}\left[\ldots F_{j+1}\left[F_{j}(P)\right]\right], G(i, j)=A_{i} \ldots A_{j}
$$

Similar in [6], we first prove

$$
E\left[\left\|P_{b_{k+1}+1}\right\| P_{b_{k}+1}=P\right]
$$

$=\sum_{j=1}^{\infty} \sum_{i=1}^{\infty}\left\|F_{b_{k}+i+j}\left[\begin{array}{l}G\left(b_{k}+i+j-1, b_{k}+i\right) \phi\left(b_{k}+i-1, b_{k}+1\right) G^{T}\left(b_{k}+i+j-1, b_{k}+i\right) \\ +\ldots A_{b_{k}+i+j-1} Q_{b_{k}+i+j-2} A_{b_{k}+i+j-1}^{T}+Q_{b_{k}+i+j-1}\end{array}\right]\right\|$

$\times(1-p)^{i-1} p(1-q)^{j-1} q=\Lambda(P)$

According to theorem 1, $P_{k+1} \leq P_{k}+C_{0}$, we can conclude

$$
\begin{aligned}
& \Lambda(P) \leq \sum_{j=1}^{\infty} \sum_{i=1}^{\infty}\left\|\left[\begin{array}{l}
G\left(b_{k}+i+j-1, b_{k}+i\right) \phi\left(b_{k}+i-1, b_{k}+1\right) G^{T}\left(b_{k}+i+j-1, b_{k}+i\right) \\
+\ldots A_{b_{k}+i+j-1} Q_{b_{k}+i+j-2} A_{b_{k}+i+j-1}^{T}+Q_{b_{k}+i+j-1}
\end{array}\right]\right\| \\
& \times(1-p)^{i-1} p(1-q)^{j-1} q+C \\
& \leq\left(\sum_{j=1}^{\infty} \sum_{i=1}^{\infty}\left\|\left[G\left(b_{k}+i+j-1, b_{k}+i\right) \phi\left(b_{k}+i-1, b_{k}+1\right) G^{T}\left(b_{k}+i+j-1, b_{k}+i\right)\right]\right\|\right. \\
& \left.+\sum_{j=1}^{\infty} \sum_{i=1}^{\infty}\left\|\left[\begin{array}{l}
G\left(b_{k}+i+j-1, b_{k}+i+1\right) Q_{b_{k}+i} G^{T}\left(b_{k}+i+j-1, b_{k}+i+1\right)+\ldots \\
A_{b_{k}+i+j-1} Q_{b_{k}+i+j-2} A_{b_{k}+i+j-1}^{T}+Q_{b_{k}+i+j-1}
\end{array}\right]\right\|\right) \\
& \times(1-p)^{i-1} p(1-q)^{j-1} q+C=\Lambda_{1}+\Lambda_{2}+C .
\end{aligned}
$$

(1) Under condition A1, we can infer:

$$
\left\|G\left(b_{k}+i+j-1, b_{k}+i\right)\right\| \leq \bar{a}^{j} \text {, }
$$

According to theorem 1 , we can infer:

$$
\phi\left(b_{k}+i-1, b_{k}+1\right) \leq \alpha\|P\|+\beta
$$

Therefore $\Lambda_{1} \leq \sum_{j=1}^{\infty} \bar{a}^{2 j}(\alpha\|P\|+\beta)(1-q)^{j-1} q$

When $\left|\bar{a}^{2}(1-q)\right|<1$, we have $\Lambda_{1}<\infty$.

$$
\Lambda_{2} \leq \sum_{j=1}^{\infty} \sum_{i=1}^{\infty}\left(\bar{a}^{2(j-1)}\left\|Q_{b_{k}+i}\right\|+\ldots \bar{a}^{2}\left\|Q_{b_{k}+i+j-2}\right\|+\left\|Q_{b_{k}+i+j-1}\right\|\right) \times(1-p)^{i-1} p(1-q)^{j-1} q .
$$

As

$$
\left\|Q_{k}\right\| \leq \bar{q} \quad, \quad \text { we }
$$
can

get $\Lambda_{2} \leq \sum_{j=1}^{\infty}\left(\sum_{j=0}^{\infty} \bar{a}^{2 j}\right) \times(1-q)^{j-1} q \bar{q}$

When $\left|\bar{a}^{2}(1-q)\right|<1$, we have $\Lambda_{2}<\infty$.

From above, we infer $E\left[\left\|P_{b_{k+1}+1}\right\| P_{b_{k}+1}=P\right] \leq \delta\|P\|+C$

Next, in the same way we can caculate

$$
E\left[\left\|P_{b_{k+1}}\right\| P_{b_{k}+1}=P\right] \leq \sum_{j=1}^{\infty} \bar{a}^{2 j}(\alpha\|P\|+\beta)(1-q)^{j-1} q+O(1)
$$

When

$$
\left|\bar{a}^{2}(1-q)\right|<1
$$
we

\section{CONCLUSION}

In this paper, we prove the bound of peak error covariance matrix of STF in the networked control system, and give the necessary and sufficient condition which guarantees the boundedness. For future work, we will compare the kalamn filter with strong tracking filter and it is of interest to find the upper and lower bound of the error covariance matrix. 


\section{REFERENCES}

[1] B. Sinopoli, L.Schenato, M. Franceschetti, K. Poolla, M. Jordan, and S. Sastry, "Kalman filtering with intermittent observations," IEEE Trans. Autom. Control [J],vol.49,no.9, 2004,pp.1453-1464

[2] Ling Shi, Michael Epstein, and Richard M. Murray. "Kalman filtering over a packet-droppping network: a probabilistic perspective", IEEE Trans Autom Control[J], vol 55 ,no.3, 2010 ,pp.594-604,

[3] Shawn Hu, Wei-Yong Yan, "Stability robustness of networked control systems with respect to packet loss", Automatica[J], vol 43,no.7, 2007, pp.1243-1248

[4] Zhou.D.H, Xi Y.G, Zhang Z.J. "extended kalman filter with suboptimal fading factor in nonlinear system", control and decision., vol5, 1990,pp1-6

[5] Konrad Reif, Stefan Gunther ,Engin Yaz, and Rolf Unbehauen. "Stochastic stability of the discrete-time extended kalman filter", IEEE Trans Autom Control[J], vol 44,no 4, 1999,pp. 714-728

[6] Minyi Huang, Subhrakanti Dey. "Stability of Kalman filtering with markovian packet losses", Automatica[J], vol .43, no.4, 2007,pp. 598-607 